Bangladesh J. Sci. Res. 29(2): 143-151, 2016 (December)

\title{
STABILITY ANALYSIS OF FINITE DIFFERENCE SCHEMES FOR AN ADVECTION DIFFUSION EQUATION
}

\author{
T. M. A. K. Azad ${ }^{*}$ and L. S. Andallah ${ }^{1}$ \\ Department of Computer Science \& Engineering, \\ University of Liberal Arts Bangladesh, Dhaka-1209, Bangladesh
}

\begin{abstract}
The paper studies stability analysis for two standard finite difference schemes FTBSCS (forward time backward space and centered space) and FTCS (forward time and centered space). One-dimensional advection diffusion equation is solved by using the schemes with appropriate initial and boundary conditions. Numerical experiments are performed to verify the stability results obtained in this study. It is found that FTCS scheme gives better point-wise solutions than FTBSCS in terms of time step selection.
\end{abstract}

Key words: Advection diffusion equation, Finite difference schemes, Stability condition

\section{Introduction}

The linear advection diffusion equation (ADE) $\frac{\partial C}{\partial t}+u \frac{\partial C}{\partial x}=D \frac{\partial^{2} C}{\partial x^{2}}$ is a model which describes the contaminant transport due to combined effect of advection and diffusion in a porous media. It is a parabolic type partial differential equation and is derived on the principle of conservation of mass using Fick's law (Socolofsky and Jirka 2002). Stability analysis of finite difference schemes for the Navier-Stokes equations is obtained (Rigal 1979). An analytical solution is obtained of the one dimensional ADE by reducing the original $\mathrm{ADE}$ into a diffusion equation by introducing another dependent variable (Al-Niami and Ruston 1977). Analytical solution of 1D ADE with variable coefficients is presented in a finite domain by using Laplace transformation technique. In that process new independent space and time variables have been introduced (Kumeret al. 2009). Numerical treatment of the mathematical model is studied for water pollution. This study was examined by various mathematical models involving water pollutant. The authors used the implicit centered difference scheme in space and a forward difference scheme in time for the evaluation of the generalized transport equation (Agusto and Bamingbola 2007). Stability and convergence in fluid flow problems is presented (Morton 1971). Stability analysis of finite difference schemes for the advection-diffusion equation is studied (Chan 1984). A comparison of some numerical methods for the advection-diffusion equation is presented (Thongmoon and Mckibbin 2006). Stability analysis of finite difference schemes for the advection diffusion equation is presented (Chan 1984). An analytical solution of the advection diffusion equation for a

*Author for correspondence: <abul.kalam@ulab.edu.bd>. ${ }^{1}$ Department of Mathematics, Jahangirnagar University, Savar, Dhaka-1342, Bangladesh. 
ground level finite area source is presented (Park and Baik 2008). An explicit finite difference scheme for solving the advection diffusion equation is studied. Numerical solution of the ADE is obtained by using FTBSCS and FTCS techniques for prescribed initial and boundary data. Numerical results for both the schemes are compared in terms of accuracy by error estimation with respect to exact solution of the $\mathrm{ADE}$ and also the numerical features of the rate of convergence are presented graphically (Azad et al. 2015).

With the above discussion in view, in the present paper, stability analysis for finite difference schemes FTBSCS and FTCS for solving the advection diffusion equation are presented. Numerical experiments are performed to verify the stability results obtained in this study. It is found that FTCS scheme gives better point-wise solutions than FTBSCS scheme in terms of time step selection.

\section{Numerical schemes for governing equation}

The volumetric concentration of a pollutant in a moving, turbulent fluid may be described by the advection-diffusion equation

$$
\frac{\partial c}{\partial t}+\nabla \cdot(u c)=D \nabla^{2} c
$$

where $c(x, y, z, t)$ is the concentration (mass per unit volume) of pollutant at point $(x, y, z)$ in Cartesian coordinates, at time $t$. The vector $u$ is the fluid velocity field and $D$ is the eddydiffusivity or dispersion tensor.

In this study, we consider one-dimensional $\mathrm{ADE}$ with constant velocity $u$ and dispersion $D$ which gives the $1-D$ advection-diffusion equation:

with initial condition

$$
\frac{\partial c}{\partial t}+u \frac{\partial c}{\partial x}=D \frac{\partial^{2} c}{\partial x^{2}}
$$

and mixed boundary conditions

$$
\begin{array}{ll}
c\left(t_{0}, x\right)=f(x) ; & a \leq x \leq b \\
c(t, a)=c_{a}(t) ; & t_{0} \leq t \leq T \\
\frac{\partial c}{\partial x}(t, b)=c_{b}(t) ; & t_{0} \leq t \leq T
\end{array}
$$

where $c_{a}, c_{b}$ are constant concentration values.

\section{Explicit finite difference scheme}

Finite difference methods involve calculating approximate values of the unknown function at a finite number of (mesh or grid) points in the domain. Here we let $0=x_{1} \leq x_{j} \leq x_{N+1}=l$ be the grid points in $x$-domain. The time is divided into equal steps of size $\Delta t$, with time $t_{n}-n \Delta t$. Derivatives are approximated by truncated Taylor Series expansions. For the numerical solution of the 1-D ADE, we consider the IBVP

$$
\frac{\partial c}{\partial t}+u \frac{\partial c}{\partial x}=D \frac{\partial^{2} C}{\partial x^{2}},
$$




$$
\begin{array}{llr}
\text { with initial condition } & c(x, 0)=f(x) & 0 \leq x<l \\
\text { and boundary conditions } & c(x=0, t)=\varepsilon_{0}, & \\
& \frac{\partial c}{\partial x}(x=l, t)=0 & 0<t \leq T
\end{array}
$$

\section{Finite difference formulae}

Derivatives in the advection diffusion equation are approximated by truncated Taylor Series expansions, which are follows:

$$
\begin{aligned}
& \frac{\partial c}{\partial t}=\frac{c_{i}^{n+1}-c_{i}^{n}}{\Delta t} \quad(1 \text { st order forward difference in time }) \\
& \frac{\partial c}{\partial x}=\frac{c_{i}^{n}-c_{i-1}^{n}}{\Delta x} \quad(1 \text { st order backward space difference formula }) \\
& \frac{\partial c}{\partial x}=\frac{c_{i+1}^{n}-c_{i-1}^{n}}{2 \Delta x} \quad(1 \text { st order centered space difference formula }) \\
& \text { and } \\
& \frac{\partial^{2} c}{\partial x^{2}}=\frac{c_{i+1}^{n}-2 c_{i}^{n}+c_{i-1}^{n}}{\Delta x^{2}}(2 \text { nd order centered space difference formula) }
\end{aligned}
$$

\section{Finite difference (FTBSCS) scheme}

Substituting equations (2), (3), (5) into equation (1) and rearranging according the time level, we get,

$$
\frac{c_{i}^{n+1}-c_{i}^{n}}{\Delta t}+u \frac{c_{i}^{n}-c_{i-1}^{n}}{\Delta x}=D \frac{c_{i+1}^{n}-2 c_{i}^{n}+c_{i-1}^{n}}{\Delta x^{2}}
$$

implies to $c_{i}^{n+1}=(\gamma+\lambda) c_{i-1}^{n}+(1-\gamma-2 \lambda) c_{i}^{n}+\lambda c_{i+1}^{n}$,

where

$$
\gamma=\frac{u \Delta t}{\Delta x}, \lambda=\frac{D \Delta t}{\Delta x^{2}}
$$

\section{Finite difference (FTCS) scheme}

Substituting equations (2), (4), (5) into equation (1) and rearranging according the time level, we get,

$$
\frac{c_{i}^{n+1}-c_{i}^{n}}{\Delta t}+u \frac{c_{i+1}^{n}-c_{i-1}^{n}}{2 \Delta x}=D \frac{c_{i+1}^{n}-2 c_{i}^{n}+c_{i-1}^{n}}{\Delta x^{2}}
$$


implies to $\quad c_{i}^{n+1}=(\gamma / 2+\lambda) c_{i-1}^{n}+(1-2 \lambda) c_{i}^{n}+\left(-\frac{\gamma}{2}+\lambda\right) c_{i+1}^{n}$,

where $\quad \gamma=\frac{u \Delta t}{\Delta x}, \lambda=\frac{D \Delta t}{\Delta x^{2}}$

It is seen that the truncation errors for the forward and backward differences are of first order; whereas the centered difference yields a second order truncation error (using by Taylor Series expansions). Therefore, both the schemes outlined above are consistent.

Also, initial condition $\mathrm{c}(\mathrm{x}, 0)=0.02 \times \mathrm{e}^{-10 \mathrm{x}}, \quad 0 \leq \mathrm{x}<1$,

and boundary conditions $\mathrm{c}(\mathrm{x}=0, \mathrm{t})=0.02$,

$$
\begin{aligned}
& \frac{\partial \mathrm{c}}{\partial \mathrm{x}}(\mathrm{x}=1, \mathrm{t})=0, \quad 0<\mathrm{t} \leq \mathrm{T} \\
& \text { implies that, } \frac{\mathrm{c}(\mathrm{n}+1, \mathrm{nx}+1)-\mathrm{c}(\mathrm{n}+1, \mathrm{nx})}{\Delta \mathrm{x}}=0 \\
& \Rightarrow \mathrm{c}(\mathrm{n}+1, \mathrm{nx}+1)=\mathrm{c}(\mathrm{n}+1, \mathrm{nx})
\end{aligned}
$$

are to be used in both the schemes stated above.

\section{Stability conditions for the FTBSCS scheme}

The explicit centered difference scheme for (1) is given by

$$
\begin{aligned}
& c_{i}^{n+1}=(\gamma+\lambda) c_{i-1}^{n}+(1-\gamma-2 \lambda) c_{i}^{n}+\lambda c_{i+1}^{n}, \\
& \text { where } \quad \gamma=\frac{u \Delta t}{\Delta x}, \lambda=\frac{D \Delta t}{\Delta x^{2}}
\end{aligned}
$$

The equation (8) implies that if

$$
\begin{aligned}
& 0 \leq \gamma+\lambda \leq 1 \\
& 0 \leq 1-\gamma-2 \lambda \leq 1 \\
& 0 \leq \lambda \leq 1
\end{aligned}
$$

then the new solution is a convex combination of the two previous solutions. That is, the solution at new time-step $(n+1)$ at a spatial node $i$ is an average of the solutions at the previous time-step at the spatial-nodes $i-1, i$ and $i+1$. This means that the extreme value of the new solution is the average of the extreme values of the previous two solutions at the three consecutive nodes. Therefore, the new solution continuously depends on the initial value $c_{i}^{0}, i=1,2,3$, .

$$
\begin{aligned}
& \text { (ii) implies } \gamma \leq 1-2 \lambda \leq 1+\gamma \ldots \ldots \ldots \text { (iv) } \\
& \text { (i) implies }-\lambda \leq \gamma \leq 1-\lambda \\
& \therefore-\lambda \leq \gamma \leq 1-2 \lambda \text { by (iv) }
\end{aligned}
$$

Therefore, the conditions are $0 \leq \lambda \leq 1$ and $-\lambda \leq \gamma \leq 1-2 \lambda$

That is $0 \leq \frac{D \Delta t}{\Delta x^{2}} \leq 1$ and $-\frac{D \Delta t}{\Delta x^{2}} \leq \frac{u \Delta t}{\Delta x} \leq 1-2 \frac{D \Delta t}{\Delta x^{2}}$. 
It is clear that $-\frac{D \Delta t}{\Delta x^{2}}$ is very small negative quantity which is physically impossible. So, we consider the stability conditions of (6) are $0 \leq \frac{D \Delta t}{\Delta x^{2}} \leq 1$ and $0 \leq \frac{u \Delta t}{\Delta x} \leq 1-2 \frac{D \Delta t}{\Delta x^{2}}$

\section{Stability conditions for the FTCS scheme}

The explicit centered difference scheme for (1) is given by

$$
c_{i}^{n+1}=(\gamma / 2+\lambda) c_{i-1}^{n}+(1-2 \lambda) c_{i}^{n}+(\lambda-\gamma / 2) c_{i+1}^{n}
$$

where $\quad \gamma=\frac{u \Delta t}{\Delta x}, \lambda=\frac{D \Delta t}{\Delta x^{2}}$

The equation (9) implies that if

$$
\begin{aligned}
& 0 \leq \gamma / 2+\lambda \leq 1 \\
& 0 \leq 1-2 \lambda \leq 1 \\
& 0 \leq \lambda-\gamma / 2 \leq 1 \text { (iii) }
\end{aligned}
$$

then the new solution is a convex combination of the two previous solutions. That is, the solution at new time-step $(n+1)$ at a spatial node $i$ is an average of the solutions at the previous time-step at the spatial-nodes $i-1, i$ and $i+1$. This means that the extreme value of the new solution is the average of the extreme values of the previous two solutions at the three consecutive nodes. Therefore, the new solution continuously depends on the initial value $c_{i}^{0}, i=1,2,3, \ldots \ldots \ldots . .$.

$$
\begin{aligned}
& \text { (ii) implies } 0 \leq \lambda \leq \frac{1}{2} \\
& \text { (iii) implies } \lambda-1 \leq \frac{\gamma}{2} \\
& \text { (i) implies }-\lambda \leq \frac{\gamma}{2} \leq 1-\lambda
\end{aligned}
$$

From (v) \& (vi), it follows that, $-\lambda \leq \frac{\gamma}{2} \leq 1-\lambda$

$$
-2 \lambda \leq \gamma \leq 2(1-\lambda)
$$

It is clear that $-\frac{D \Delta t}{\Delta x^{2}}$ is very small negative quantity which is physically impossible. So, we consider the stability conditions of (7) are

$$
0 \leq \frac{D \Delta t}{\Delta x^{2}} \leq \frac{1}{2} \text { and } 0 \leq \frac{u \Delta t}{\Delta x} \leq 2\left(1-\frac{D \Delta t}{\Delta x^{2}}\right)
$$

\section{Numerical results and discussions}

Various finite difference equations were used to approximate the parabolic model equation (1). It is interesting to experiment with these numerical techniques. It is hoped that by writing computer codes and analyzing the results, additional insights into the solution procedures are gained. Therefore, this section proposes an example and presents solutions by the described schemes. 


\section{Problem description}

In this study, we assume that spatial length, $l=18 \mathrm{~m}, D=0.01 \mathrm{~m}^{2} / \mathrm{s}=36 \mathrm{~m}^{2} / \mathrm{h}, u=0.01 \mathrm{~m} / \mathrm{s}=36 \mathrm{~m} / \mathrm{h}$ ,$\Delta x=0.05$ mat all time, $t=1$ minute to $t=6$ minutes.

The one-dimensional advection diffusion equation for this problem $\frac{\partial C}{\partial t}+u \frac{\partial C}{\partial x}=D \frac{\partial^{2} C}{\partial x^{2}}$ is to be solved with initial condition and boundary conditions

$$
\begin{array}{ll}
c(x, 0)=0.02 \times e^{-10 x}, & 0 \leq x<l \\
c(x=0, t)=0.02, & 0 \leq t<T \\
\frac{\partial c}{\partial x}(x=l, t)=0 &
\end{array}
$$

Various values of time steps are to be used to investigate the numerical schemes and the effect of steps on stability.

\section{Results and Discussion}

Case I. In this case, both schemes are to be used as stated previously:

The stability requirements of the FTBSCS scheme are

$0 \leq \frac{D \Delta t}{\Delta x^{2}} \leq 1$ and $0 \leq \frac{u \Delta t}{\Delta x} \leq 1-2 \frac{D \Delta t}{\Delta x^{2}}$ (The terms $\frac{u \Delta t}{\Delta x}=\gamma$ and $\frac{D \Delta t}{\Delta x^{2}}=\lambda$ are known as the advection number and diffusion number, respectively.)

When the time step is increased to $\Delta t=0.1$,

For this particular application, $\lambda=\frac{D \Delta t}{\Delta x^{2}}=\frac{0.01 \times 0.1}{(0.05)^{2}}=0.4$

$$
\begin{gathered}
\gamma=\frac{u \Delta t}{\Delta x}=\frac{0.01 \times 0.1}{0.05}=0.02 \\
\therefore \frac{D \Delta t}{\Delta x^{2}}=\frac{0.01 \times 0.1}{(0.05)^{2}}=0.4 \leq 1 \text { and } 0 \leq 0.02 \leq 0.2
\end{gathered}
$$

And the stability requirements of the FTCS scheme are

$$
0 \leq \frac{D \Delta t}{\Delta x^{2}} \leq \frac{1}{2} \text { and } 0 \leq \frac{u \Delta t}{\Delta x} \leq 2\left(1-\frac{D \Delta t}{\Delta x^{2}}\right) .
$$

For this particular application,

$$
\therefore \frac{D \Delta t}{\Delta x^{2}}=\frac{0.01 \times 0.1}{(0.05)^{2}}=0.4 \leq \frac{1}{2} \text { and } 0 \leq 0.02 \leq 1.20
$$

Therefore, the stability condition is satisfied, and a stable solution is expected. The concentration profiles for both the schemes are to be obtained up to $t=6$ minutes are shown in Fig. 1. 


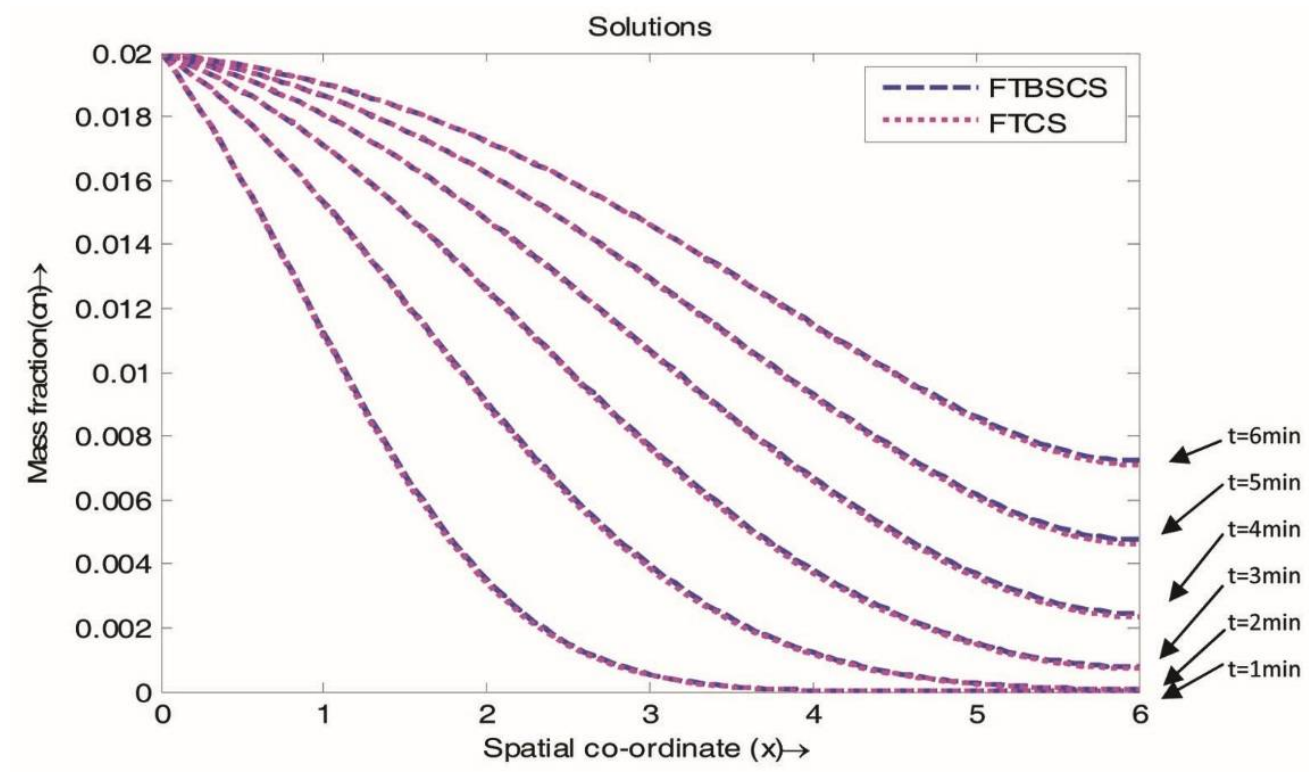

Fig. 1. Concentration profiles with $\Delta x=0.05, \Delta t=0.1$.

\section{Case II:}

When the time step is increased to $\Delta t=0.122$, which is only a fraction of an increase over preceding cases.

In this case, the stability conditions of FTBSCS scheme are

$$
0 \leq \frac{D \Delta t}{\Delta x^{2}} \leq 1 \text { and } 0 \leq \frac{u \Delta t}{\Delta x} \leq 1-2 \frac{D \Delta t}{\Delta x^{2}} .
$$

For this particular application, $\lambda=\frac{D \Delta t}{\Delta x^{2}}=\frac{0.01 \times 0.122}{(0.05)^{2}}=0.488$

$$
\gamma=\frac{u \Delta t}{\Delta x}=\frac{0.01 \times 0.122}{0.05}=0.0244
$$

$\therefore \frac{D \Delta t}{\Delta x^{2}}=\frac{0.01 \times 0.122}{(0.05)^{2}}=0.488 \leq 1$ and $0 \leq 0.0244 \leq 0.024$, which exceeds the stability requirement.

And the stability requirements of the FTCS scheme are

$$
0 \leq \frac{D \Delta t}{\Delta x^{2}} \leq \frac{1}{2} \text { and } 0 \leq \frac{u \Delta t}{\Delta x} \leq 2\left(1-\frac{D \Delta t}{\Delta x^{2}}\right)
$$

For this particular application,

$$
\therefore \frac{D \Delta t}{\Delta x^{2}}=\frac{0.01 \times 0.122}{(0.05)^{2}}=0.488 \leq \frac{1}{2} \text { and } 0 \leq 0.0244 \leq 1.024 \text {. }
$$


Therefore, at this stage one of the stability conditions for FTBSCS is not satisfied, and an unstable solution is appeared. With the step sizes indicated, an unstable solution is developed. The velocity profiles are to be obtained at $t=6$ minutes are shown in Figure 2 .

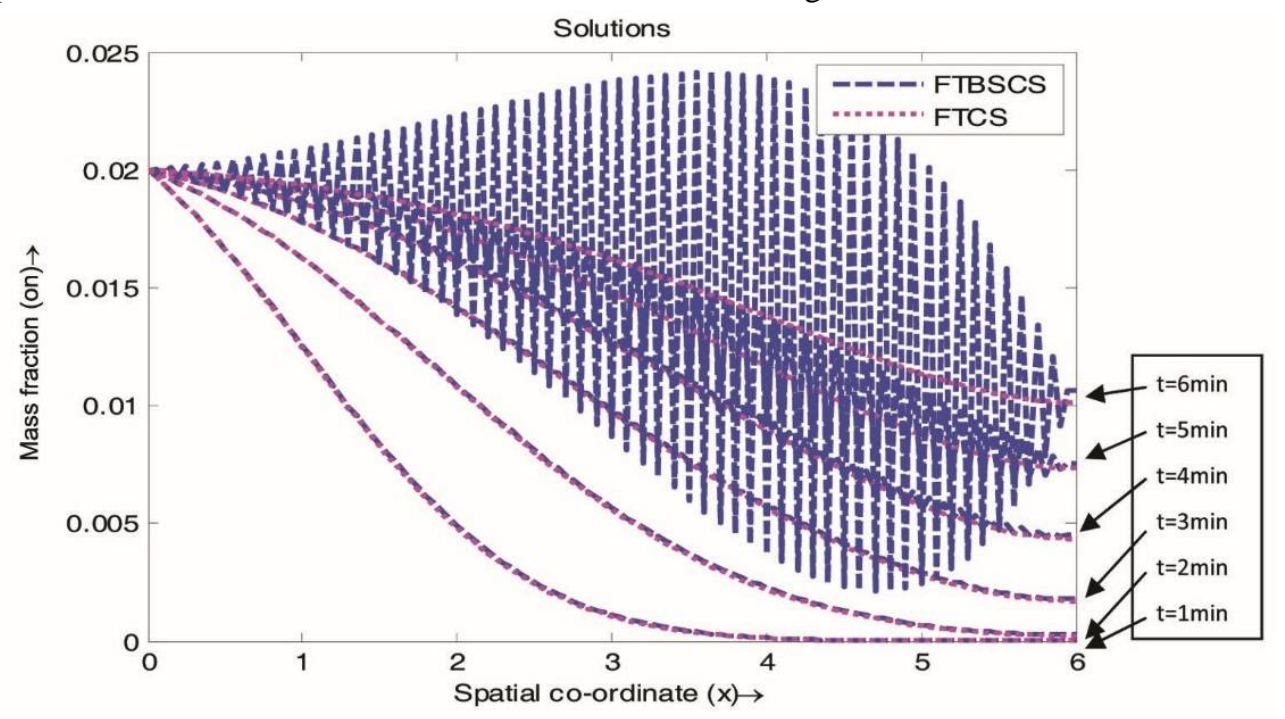

Fig. 2. Concentration profiles with $\Delta x=0.05, \Delta \mathrm{t}=0.122$

\section{Conclusion}

Authors have presented stability analysis and numerical solutions by using FTBSCS and FTCS schemes for 1D advection diffusion equation with an initial condition and two boundary conditions. Numerical experiment is presented graphically. In the Fig. 1, it has been found that FTCS scheme gives better pointwise solution than FTBSCS. Fig. 2, an unstable solution is appeared by using FTBSCS scheme however, the solutions by using FTCS scheme is stable at the increased time step size $\Delta \mathrm{t}=0.122$ and it is shown that FTCS scheme is superior to FTBSCS scheme interms of time step selection.

\section{References}

Agusto, F.B. and O.M. Bamingbola. 2007. Numerical treatment of the mathematical models for water pollution. Res. J. Appl. Sci. 2(5): 548-556.

Al-Niami, A.N.S and K.R.Ruston 1977. Analysis of flow against dispersion in porous media J. Hydrol. 33: $87-97$.

Azad, T.M.A.K., M. Begum and L.S.Andallah. 2015. An explicit finite difference scheme for advection diffusion equation 2015. Jahangirnagar J. Mathematics and Mathematical Sciences 24: 2219-5823.

Chan, T. F. 1984. Stability analysis of finite difference schemes for the advection diffusion equation. SIAM J. Numer. Anal. 21: 272-284.

Kumar, A., D. K. Jaiswal and N. Kumar. 2010. Analytical solution to one dimensional advection diffusion equation with variable coefficients in semi-infinite media. J. Hydrol. 380: 330-337. 
Kumar, A., D. K. Jaiswal and N. Kumar. 2009. Analytical solution of one dimensional advection diffusion equation with variable coefficients in a finite domain. J. Earth Syst. Sci.118: 539-549.

Morton, K.W. 1971. Stability and convergence in fluid flow problems. Proc. Roy. Soc. London A. 323: 237-253.

Park, Y.S., J. J. Baik. 2008. Analytical solution of the advection diffusion equation for a ground level finite area source. Atmospheric Environment 42: 9063-9069.

Rigal, A. 1979. Stability analysis of explicit finite difference schemes for the Navier-Stokes equations. Internat. J. Numer. Math. Engng. 14: 617-628.

Socolofsky, S. A. and G.H. Jirka. 2002. Environmental Fluid Mechanics. Part 1, 2nd Edition, Institute for Hydrodynamics, University of Karlsruhe, Germany.

Thongmoon, M. and R. Mckibbin. 2006. A comparison of some numerical methods for the advectiondiffusion equation. Int. Math. Sci. 10:49-52.

(Manuscript received on 21 October, 2016; revised on 8 January, 2017) 